\title{
Feature-Centric Image Enhancement via Dehazing
}

\author{
Prince Owusu-Agyeman ${ }^{1 *}$, Xie Wei ${ }^{1}$, Yao Yeboah ${ }^{2}$ \\ ${ }^{1}$ School of Automation Science and Engineering, South China University of Technology, Guangzhou, P.R. \\ China. \\ ${ }^{2}$ School of Automation Science and Engineering, Guangdong University of Technology, Guangzhou, P.R. \\ China.
}

* Corresponding author. Tel.: +8613450461031; email: princeowusu-agyeman@outlook.com Manuscript submitted December 20, 2017; accepted March 11, 2018.

doi: 10.17706/ijcee.2018.10.2.146-157

\begin{abstract}
In this paper, we address the image dehazing problem through a global feature-restoration pipeline. We propose a dark channel prior-based global image dehazing algorithm which captures and restores the true features of pixels within haze-degraded regions by applying scene depth selection and adaptive filtering. Our scheme harnesses haze and depth features intuitively across a given image without the prior scene depth information. This allows our scheme to sustain a high dehazing efficiency across all image regions irrespective of the local depth variations. We prove that haze degradation is linearly correlated with scene depth and based on this nuance, propose a depth selection and cropping scheme, which guides the adaptive filter iteratively across the image. Secondly, we put forward haze relevant image features and highlight the dark-channel prior for image dehazing. We merge the dark channel prior and scene depth-cropping schemes into a unified dehazing pipeline which is capable of sustaining uniform and robust results across all image regions, in real-time. We verify the superiority of the proposed scheme in terms of speed and robustness through computer-based experiments. Finally, we present comparison results with state-of-the-art and further highlight the comparative superiority of our scheme.
\end{abstract}

Key words: Dark channel prior, real-time dehazing, feature restoration, image enhancement.

\section{Introduction}

In digital image processing and other areas of pattern recognition, machine vision systems and related applications, image corruption continues to remain a core challenge. Most outdoor imaging systems and applications such as security surveillance [1], remote sensing [2], autonomous and self-driving technologies [3], traffic monitoring [4] and other related real-time computer visions systems, are adversely affected by this multi-facetted image corruption process. While various conditions may lead to image corruption in real-time applications, adverse weather condition has been strongly linked with this degradation in outdoor applications [5]. Under the uncontrolled conditions of outdoor applications, the image acquisition process is easily impacted upon by poor weather conditions including but not limited to fog, haze and smoke particles. For most real-time systems, feature extraction is a crucial mid-level operation that seeks to construct certain descriptors such as Histogram Oriented Gradients (HOG), Scale Invariant Feature Transform (SIFT), Wavelet transform coefficients but to mention a few. Such features are usually the essential fundamentals upon which higher level machine learning schemes can be realized. Left unaddressed, the degradation of acquired images as a result of haze could lead to local and global feature 
corruption, a process which has manifold adverse impacts upon overall system performance.

The corruption process associated with images acquired under hazy conditions has been well established by some existing works including but not limited to [6]-[8]. This forward degradation process is formulated as:

$$
I(x)=J(x) t(x)+A(1-t(x)) .
$$

In the Equation (1), $A$ represents the global atmospheric light, $I$ represents the intensity captured by the acquisition system, $J$ is the scene radiance and finally, $t$ represents the transmission of the medium. The goal of image dehazing is therefore formulated as the successful recovery of the $J$, $t$, and $A$ parameters from the acquired image, $I$. Numerous schemes have been previously proposed towards image enhancement via dehazing including but not limited to Retinex [9], Guided Filters [10], Wavelet based fusion [11] and analytical color modeling [12]. Most of such early schemes have relied extensively on multi-image prior schemes and fail when such requirements are unsatisfied. In [13]-[15], multiple constraints are enforced using images from varying conditions. Such constraints may not always be satisfied, especially in real-time systems when conditions are not known before-hand. The Dark-Channel prior originally proposed in [16], opened new possibilities for single image dehazing. This prior offered a sound statistical approach to image dehazing which allowed for the dehazing task be realized using single images. Despite its significant extensions over recent years [17]-[19], the scheme still remains constrained to local patch-based schemes which fail to capture the global color composition of the image. This has led to the color distortion problem associated with dehazing outputs, a current problem in state-of-the-art. We address the image dehazing problem as a global color feature restoration process. Our scheme therefore harnesses haze pixel features across an entire image without constraining the problem to local patch-based schemes but extending efficiency to distant regions within the scene as well. We propose a dark channel prior-based global image dehazing algorithm which captures and restores the true features of pixels within haze degraded regions by iterating the input sequence using a depth-based selection scheme. Since the haze degradation process is correlated with the depth of the scene, this depth-based selection scheme allows for the scene priors to be efficiently selected and applied in robust global dehazing. Our scheme is therefore capable of extending performance to distant regions within the image, and contrary to most state-of-the-art, dehazing performances does not degrade in distant scene patches.

\section{Previous Work}

\subsection{Problem Statement}

Image enhancement by means of dehazing has been thoroughly researched over the years. We conduct a brief background review of some key contributions which relate to the approach taken to the dehazing problem in this paper.

Perhaps the atmospheric scattering model has established one of the most efficient means of formulating and decomposing the haze formation process. Initially proposed in [20] and further extended over the years in [14] and [15], the atmospheric scattering model can be generally expressed as represented in the Eq. (1). In the Eq. (1), $t(x)$ represents the component of light that makes it to the acquisition device and can be formulated as:

$$
t(x)=e^{(-\beta d(x))} .
$$

In the Eq. (2), $d(x)$ represents the distance between a point of interest within the target haze scene and the acquisition device. The scattering coefficient of the medium is represented as $\beta$. While the formulation 
of Eq. (2) suggests that the distance, $d(x)$ yields $A=I(x)$ as it approaches infinity, practical scenarios constrain this distance to practical, albeit large, real numbers. This distance possesses an inverse relationship with the transmission parameter, $t_{0}$, thereby establishing one of the core problems of image dehazing; successfully recovering image features within distant scene regions. Once the atmospheric scattering coefficient, $A$, and the scene transmission, $t$, have been determined, the true scene is obtainable using the formulation:

$$
J=\frac{1-A}{t}+A
$$

\subsection{Haze-Relevant Features}

Real-time image dehazing is an ill-posed problem, the nature of which has continually encouraged the design of prior information towards a successful solution. Over the years, multiple prior assumptions have been proposed towards the successful deduction of so-called haze-relevant features, crucial to successful dehazing.

- Local Maximum Contrast

It is deducible from the Eq. (1) that the contrast of the acquired image is impacted upon by the haze transmission $(t \leq 1)$ as:

$$
\sum_{x}\|\nabla I(x)\|=t \sum_{x}\|\nabla J(x)\| \leq \sum_{x}\|\nabla J(x)\| .
$$

Drawing from this observation, the work in [21] proposes a local contrast enhancement scheme. Within a local patch, $r x r$, the local contrast is defined as the variance of the pixel intensities within given patch as compared to the center pixel. This can be formulated as:

$$
C(x)=\max _{y \in \Omega_{r}(x)} \sqrt{\frac{1}{\left|\Omega_{s}(y)\right|} \sum_{Z \in \Omega_{s}(y)}\|I(Z)-I(y)\|^{2}} .
$$

In the Eq. (5), the parameter $\left|\Omega_{s}(y)\right|$ represents the cardinality associated with the local neighborhood $\left|\Omega_{s}(y)\right|$. The scene visibility is therefore recoverable through the maximization operation presented in the Eq. (5).

- Hue Disparity

The work in [22] has proposed to detect and estimate haze through a computation of the hue disparity between the original image, $I(x)$ and its semi-inverse, $I_{s i}(x)$. This semi-inverse image is obtainable through max $\left[I^{c}(x), \mathrm{P}-I^{c}(x)\right]$, where $c \in\{r, g, b\}$. This Hue disparity feature is then formulated as:

$$
H(x)=\left|I_{s i}^{h}(x)-I^{h}(x)\right| .
$$

In the Eq. (6), $h$ denotes the hue channel associated with the image within the HSV color space. The medium transmission $t(x)$ maintains an inverse relationship with $H(x)$.

- Color Attenuation

The color attenuation feature for image dehazing has been established based on the observation that the saturation, $I^{s}(x)$ of a patch within the image decreases abruptly under the impact of haze, while inversely, the brightness, $I^{v}(x)$, increases at the same time, yielding higher values. This color attenuation feature 
originally presented in [23], measures the difference between the brightness and saturation which produces a measure of the haze concentration within the local patch as:

$$
A(x)=I^{v}(x)-I^{s}(x) .
$$

The parameters $I^{v}(x)$ and $I^{s}(x)$ are expressible within the HSV color space as $I^{v}(x)=\max _{c \in\{r, g, b\}} I^{c}(y)$ and $I^{s}(x)=\left(\max _{c \in\{r, g, b\}} I^{c}(x)-\min _{c \in\{r, g, b\}} I^{c}(x)\right) / \max _{c \in\{r, g, b\}} I^{c}(x)$. The color attenuation has been shown to enable straight-forward computation of the medium transmission and has established a strong prior towards efficient single-image dehazing.

- Multi-Scale Local Max Saturation

Quite similarly to the local image contrast feature, it has been established in previous work that the image saturation is also affected by haze. The local maximum saturation, defined as the maximum pixel-wise saturation within a local $r x r$ patch can therefore be treated as a haze-relevant feature and formulated as:

$$
S_{r}(x ; I)=\max _{y \in \Omega_{r}(x)}\left(1-\frac{\min _{c \in\{r, g, b\}} I^{c}(y)}{\max _{c \in\{r, g, b\}\}^{c}(y)}}\right) .
$$

- Multi-Scale Dark Channel

Drawing from the deficiencies of earlier haze-related features, the dark-channel prior was proposed in [16]. This statistical prior is based on the observation on a wide observation of haze-free outdoor images which stipulates that in most haze-free patches, at least one of the color channels has associated pixels with intensities close or equal to zero. The prior is then defined as the minimum of all pixel colors within a given local patch:

$$
D(x)=\min _{y \in \Omega_{r}(x)}\left(\min _{c \in\{r, g, b\}} I^{c}(y)\right)
$$

where $I^{c}$ represents the RGB color channel associated with $I$ and $\Omega_{r}(x)$ represents the local patch which is centered around $x$ with a corresponding size of $r x r$. This haze-related feature has been shown to demonstrate a strong correlation with the haze concentration within a given image. Furthermore, it allows for a direct estimation of the medium transmission such that $t(x) \propto 1-D(x)$.

Perhaps apart from prior-based feature schemes, another major contribution in the single image dehazing is the Retinex Theory [9]. the Retinex theory is based on the dominant assumption that any given image can be decomposed into reflectance and illumination. These assumptions establish the foundation upon which the design of image dehazing techniques that are capable of adjusting the perceived quality of the image from a viewer's point of view can be realized. Some classical works in the area are the Single-Scale Retinex (SSR) [24] and the Multi-Scale Retinex (MSR) [25]. A core drawback with such schemes however is their tendency to over-fit the reflectance feature, resulting in outputs that appear highly artificial and unnatural. The work presented in this paper however does not suffer such drawbacks. Compared with most state-of-the-art, our scheme is capable of achieving real-time and efficient dehazing results that have the capability of attaining global feature boosting and therefore establishing the foundation for more robust feature extraction and other higher level vision and learning operations such as object detection, tracking and recognition. The remainder of the paper is thus organized. In the Section 3, We present the proposed real-time dehazing framework and establish the correlation between the depth feature and haze intensity. The theoretical and mathematical constitution of the algorithm is presented and discussed. Section 4 presents experimental verification and evaluation results. We further compare the performance of 
our proposed scheme with state of the art. The paper concludes in Section 5.

\section{Proposed Real-Time Dehazing Scheme}

\subsection{Establishing the Correlation between Scene Depth and Haze Intensity}

The correlation between haze intensity and scene depth establishes a context within which haze formation can be better understood and modeled. The atmospheric scattering model establishes that the overall light reaching the image acquisition system is a combination of incident light and atmospheric radiation [26]. This can be formulated as:

$$
I(x)=I_{\infty} \rho e^{-\beta d}+I_{\infty}\left(1-e^{-\beta d}\right) .
$$

Within the Eq. (10), the component $I_{\infty}$ represents the environmental radiation intensity, and $\rho$ is the scene reflectance. Likewise, $\beta, \mathrm{d}$ and $I_{\infty} \rho$ represent the scattering coefficient, field depth and scene radiance respectively. Finally, the component, $e^{-\beta d}$ represents the un-attenuated light component which propagates the medium and is captured at the acquisition point. From the expression, it is clear that the parameter decreases with increase in the field depth, $d$. The attenuated component of light is represented by the expression $I_{\infty} \rho e^{-\beta d}$. We can notice that attenuation increases with an increase in the depth of field and this results in lower contrasts in distant regions of the scene. This phenomenon is depicted in the Fig. 1 below.

Having established this correlation between scene depth and haze intensity, it therefore becomes clear that there is the need for introducing a new parameter into the Eq. (11), which would allow us to approximate the attenuation towards an average within a local depth patch. By assuming that the attenuation remains constant for all color channels, we introduce this control parameter as:

$$
I(x)=I_{\infty}\left(1-e^{-\beta d}(\min (R G B))\right) .
$$
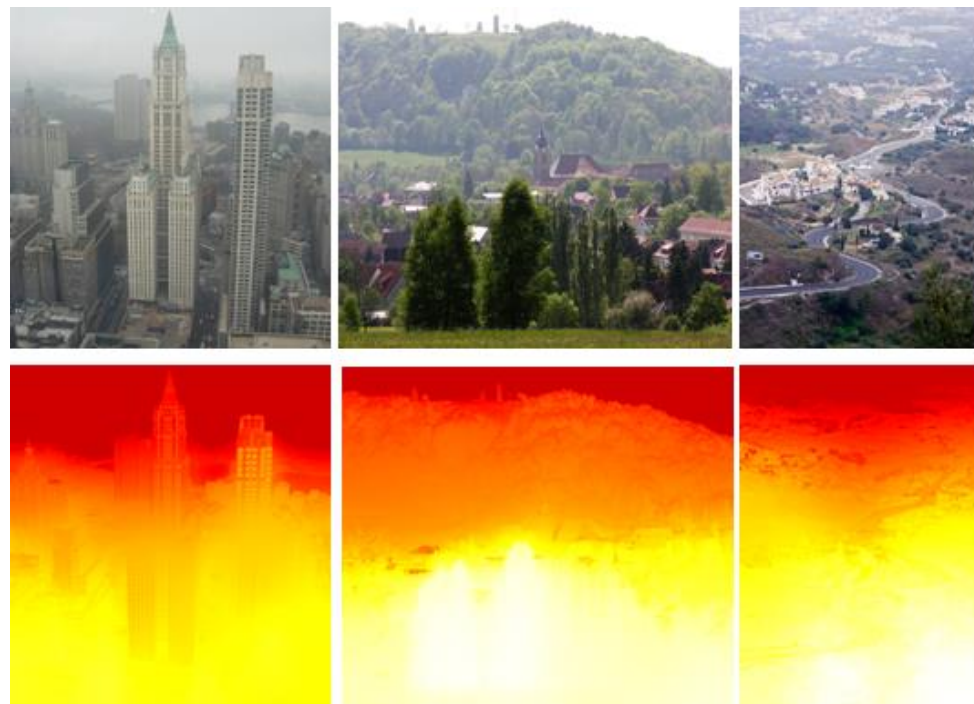

Fig. 1. An illustration of the correlation between depth and haze intensity. Top: Input haze image, bottom: depth map demonstrating scene in depth segments.

By relying on this new formulation expressed in the Eq. (11), we produce a scheme by which global image dehazing can be performed through an iterative patch-based scheme, relying on the local pixel depths through the $\min (R G B)$ parameter. 


\subsection{Depth-Guided Dehazing for Global Feature Enhancement}

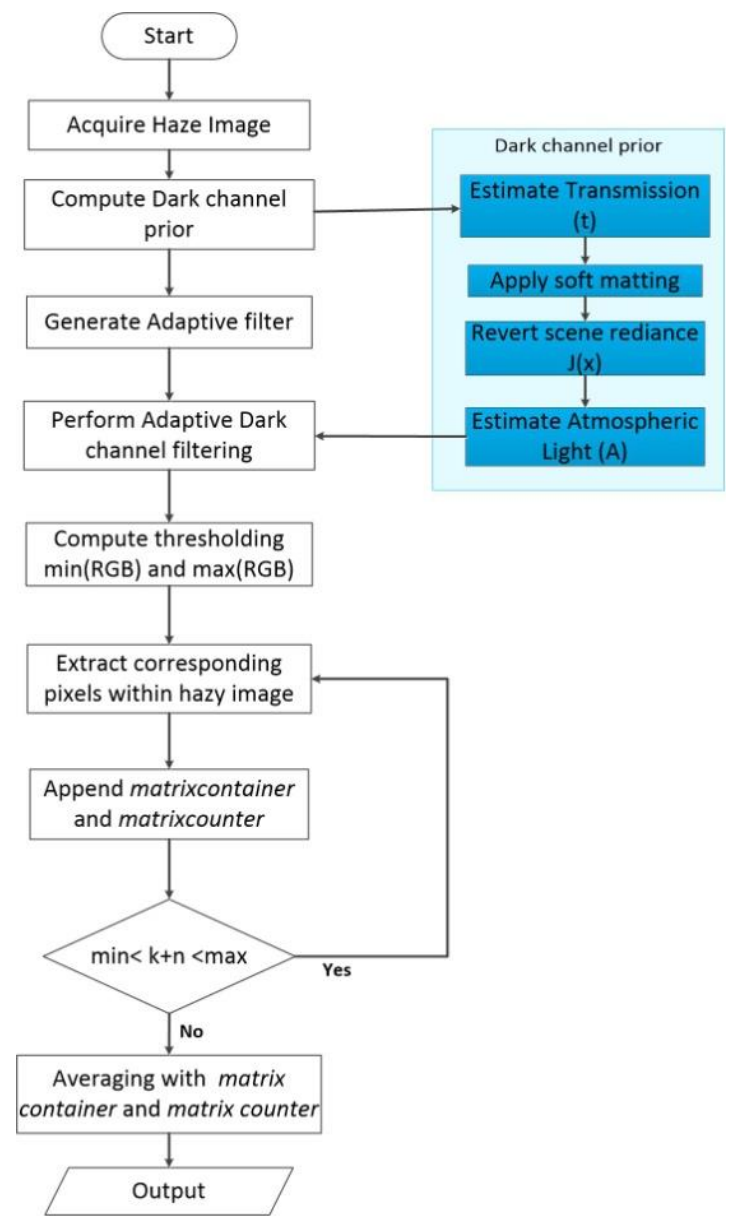

Fig. 2. An illustration of the processing pipeline of the proposed dehazing algorithm.

The overall framework of the proposed dehazing scheme is illustrated in the Fig. 2 below. As previously stated, our scheme, while based on the Dark channel prior, further takes advantage of adaptive filtering schemes in further enhancing dehazing effects which are uniformly efficient across all depth segments within the target image. Using the depth as a tool for guiding the algorithm, the proposed scheme is capable of selecting upper and lower depth threshold values for defining a specific image region for dehazing. In an iterative manner, this scheme is capable of traversing an entire haze input image and achieving superior dehazing effects, as we present in the Section 4 below. Firstly, the Dark Channel prior is obtained from a given image. After this is achieved, we compute a minimization of the given input image in RGB space. At this point, the lower and upper RGB thresholds which we denote as $\min (R G B)$ and $\max (R G B)$. We then obtain a fixed value within the image pixels with a given length $l$ and a stepping value $n$. Based on these parameters, we proceed to extract the pixels within the hazy image that fall within the range determined to be attributed to haze pixels. The pixels extracted in the previous step are summed up into a single matrix, matrixcontainer and a parameter matrixcounter is incremented by the value 1 for each iteration. We rely on the step value, $n$, to ensure that $k+n$ remains above the min and below the max threshold values. While this condition holds true, we continue to perform pixel extraction operations. Once all iterations are exhausted, the algorithm proceeds to the final stage where pixel balancing is performed using the matrixcontainer and matrixcounter for performing an averaging across all extracted pixel values. This provides a final value for achieving global dehazing across the entire image. 


\section{Experimental Verification and Evaluation Results}

In this section of the paper, we present verification of the proposed algorithm using multiple metrics and further perform some comparison experiments with state-of-the-art. In order to ensure fairness in comparison, all algorithms are evaluated in the same Matlab software environment. The test bed platform for performing this experimental evaluation has the following hardware features: Pentium quad core processor at $2.8 \mathrm{GHz}$ with a $6 \mathrm{~GB}$ internal memory. Since the goal of a majority of dehazing schemes is to improve the visual quality of images as well as boost intrinsic image features for higher level processing operations, two categories of experimental results are presented here in this section. Firstly, we present qualitative experimental results aimed at highlighting the visual improvements achieved with the proposed scheme. The second category of results is presented from quantitative experiments which aim at highlighting the feature improvement benefits of the proposed dehazing method. Finally, we present some computational complexity and speed metrics in order to highlight the real-time feasibility of the proposed method. All results are presented in comparison with state-of-the-art.

\subsection{Qualitative Visual Evaluation}

As illustrated in Fig. 3, the proposed dehazing scheme does not only perform efficiently in restoring the visual quality of the image within the RGB channels, but also, depth information within the scene is significantly improved. Additionally, by harnessing a global approach to addressing dehazing, the algorithm's efficiency does not become constrained to nearby patches within the image but the same efficiency is uniformly extended to distant regions within the image. The capability of the proposed scheme to efficiently reconstruct scene depth has strong implications for real-time systems since for most systems, depth information is immensely relied upon in achieving object segmentation which allows for the realizing of several higher level operations such as target classification, recognition and tracking.

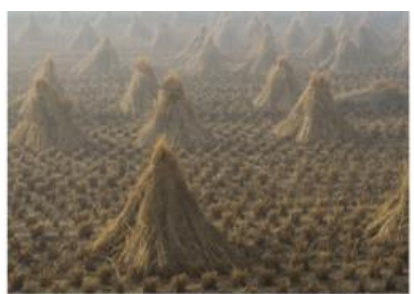

(a)

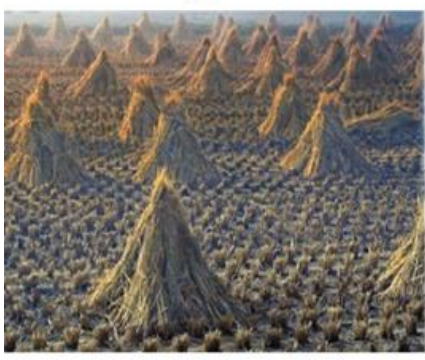

(c)

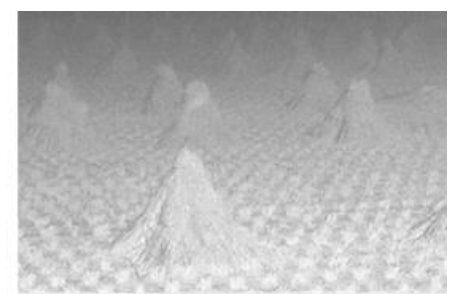

(b)

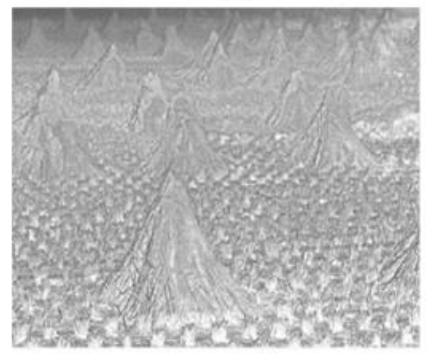

(d)

Fig. 3. The proposed dehazing scheme effectively reconstructs the scene transmission, essentially restoration depth information within the scene (a) Input haze image (b) Transmission map of haze image (c) Dehazed image (d) Transmission map of dehazed image.

The Fig. 4 presents qualitative comparison results with the methods presented in [16], [23], [27], [28]. The attained results demonstrate the superiority of the proposed scheme in comparison the state-of-the-art in several instances. Firstly, due to an overestimation of the atmospheric light, the methods presented in [16] and [28] tend to produce dehazing outputs with several discolored patches, especially in sky regions within 
the target image. This drawback is intrinsic to several dark channel based methods that fail to apply more intuitive schemes in determining the atmospheric light; a crucial parameter in image dehazing. While the method presented in [23] overcomes the overestimation drawback, it is noticeable that the achieved dehazing results on several images from the dataset comes riddled with color infidelities. That is to say that the reconstructed images tend to have local regions with high dehazing effects but from a global scope, these patches tend to have very high contrasts, producing dehazing outputs with unnatural visual image properties. Furthermore, for image with high depth ranges, dehazing results are only effective for close-ranged patches while a significant volume of haze continues to remain in distant regions. We associate this drawback with the lack of effective depth-modeling strategies which are capable of intuitively adapting parameters for the various depth patches within the image. This has several adverse effects on higher level machine vision or learning schemes which may only rely on single images from the scene for the realization of feature extraction. Finally, due to the rigidity in filtering that is applied in some of the state-of-the-art, some results may end up with smoothing effects that may remove certain crucial edge and boundary features. In contrast with the state-of-the-art, the proposed method achieves results with high levels of visual clarity and color fidelity which remain uniform across varied depth regions of the image.

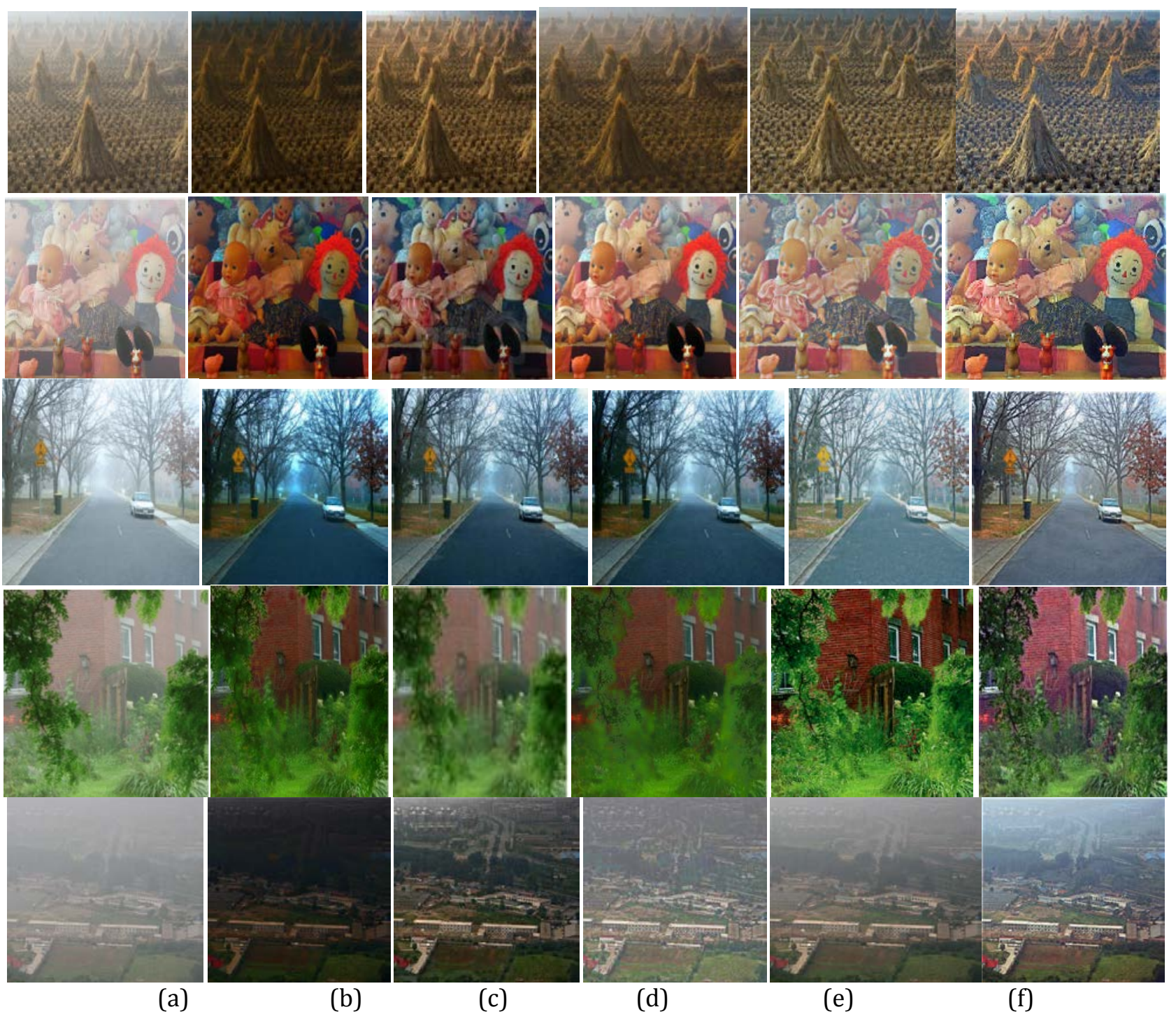

Fig. 4. Visual comparison of various state-of-the-art dehazing algorithms and the proposed scheme where (a) original image (b) [16] (c) [28] (d) [23] (e) [27] (f) proposed scheme. 


\subsection{Quantitative Evaluation and Experiments}

As previously stated, image dehazing does not only seek to reconstruct images in order to provide visual clarity but also to reconstruct intrinsic image properties, which are crucial for high level machine vision and learning processes. In order to demonstrate the superiority of the proposed feature-centric dehazing method in comparison with state-of-the-art, we present quantitative experimental results in this section. The metrics relied upon in presenting the quantitative comparison results are, Mean Squared Error (MSE), Peak Signal to Noise Ratio (PSNR), Signal to Noise Ratio (SNR) and Structural Similarity Index Measure (SSIM).

A core challenged encountered in the performance of such quantitative comparison experiments lies in the fact that the computation of the fore-mentioned metrics requires both dehazed output image as well as a reference image in the form of the original image prior to haze corruption. The challenge associated with this approach is the lack of datasets which contain the original and accompanying haze image pairs. In overcoming this challenge, we select several haze-free images from the internet and perform artificial haze synthesis using a synthesis model in order to generate the requisite haze-free and dehazed image pairs. The Table 1 below presents the quantitative comparison results with state-of-the-art. The table presents average values for each algorithm out of a total of 50 experiments.

Table 1. A Comparison of the Proposed Algorithm with State-of-the-Art (Intrinsic Feature Restoration)

\begin{tabular}{lllll}
\hline \hline Algorithm & MSE & PSNR & SNR & SSIM \\
\hline He et al.[16] & 4246.58 & 12.26 & 6.82 & 0.61 \\
He et al.[28] & 1398.47 & 16.86 & 11.57 & 0.68 \\
Tarel et al.[27] & 2324.43 & 14.6 & 9.37 & 0.72 \\
Lai et al.[23] & 1866.3 & 15.6 & 10.58 & 0.67 \\
Proposed & 1066.69 & 17.99 & 12.7 & 0.75 \\
& & & &
\end{tabular}

The quantitative results achieved clearly highlight the superiority of the proposed scheme on all metrics. This demonstrates the high reconstruction capabilities of the proposed scheme not only for boosting the visual quality of images but also for effectively enhancing the intrinsic image features upon which high level schemes rely. In contrast to the superiority of the proposed scheme, the most inferior performance is attained by the method proposed in [16]. Finally, we present computational complexity comparisons in the Table 2 .

Table 2. A Comparison of the Proposed Algorithm with State-of-the-Art (Computational Time)

\begin{tabular}{ll}
\hline \hline Algorithm & Computational Overhead (s) \\
\hline He et al.[16] & 29.5 \\
He et al.[28] & 619.5 \\
Tarel et al.[27] & 4.7601 \\
Lai et al.[23] & 147.6 \\
Proposed & 8.4 \\
& \\
\hline \hline
\end{tabular}

In order to keep up with real-time system requirements, there is the need to address the computational complexities of dehazing methods. The attained results suggest that the proposed method, while superior in performance, maintains impressive computational speeds with very low computational overheads which are only outperformed by the results attained by the method presented in [27]. This highlights a possible trade-off that is to be considered in current dehazing schemes between speed and efficiency. This trade-off is an interesting topic for future research efforts. 


\section{Conclusion}

The work in this paper addresses image dehazing as a global feature restoration process. We harness pixel level information as well as scene depth as strong emphasis upon which dehazing is performed. The scheme, which is based on the dark channel prior is capable of capturing and restoring the true features of pixels within even distant regions of the target hazy image. We establish a strong correlation between scene depth and haze intensity, then proceed to present our feature-centric dehazing algorithm for real-time applications. We demonstrate that by efficiently tying-in depth information into the dehazing framework, an increase in depth within the scene does little to hamper the dehazing efficiency of the proposed scheme. Furthermore, we perform qualitative and quantitative experimental verifications and comparisons with state-of-the-art and show that our scheme does not only outperform the state-of-the-art in terms of speed, but is also more successful in reconstructing the intrinsic image features and bringing them significantly closer to the original, haze-free images. Future work will focus on the extension of this work using machine learning to train an agent with haze-relevant features, and then proceeding to apply this learned agent in online end-to-end feature-centric dehazing.

\section{Acknowledgment}

The authors would like to express their appreciation to the 303 research lab of the School of Automation Science and Engineering, South China University of Technology.

\section{References}

[1] Puvvadi, U. L. N., Benedetto, K. D., Patil, A., Kang, K. D., \& Park, Y. (2015). Cost-effective security support in real-time video surveillance. IEEE Transactions on Industrial Informatics, 11(6), 1457-1465.

[2] Xie, F., Shi, M., Shi, Z., Yin, J. \& Zhao, D. (2017). Multilevel cloud detection in remote sensing images based on deep learning. IEEE Journal of Selected Topics in Applied Earth Observations and Remote Sensing, 99, 1-10.

[3] Lee, U., Jung, J., Shin, S., Jeong, Y., Park, K., Shim, D. H., \& Kweon, I. (2016). EureCar turbo: A self-driving car that can handle adverse weather conditions. Proceedings of the 2016 IEEE/RSJ International Conference on Intelligent Robots and Systems (IROS) (pp. 2301-2306).

[4] Datondji, S. R. E., Dupuis, Y., Subirats, P. \& Vasseur, P. (2016). A Survey of vision-based traffic monitoring of road intersections. IEEE Transactions on Intelligent Transportation Systems, 17(10), 2681-2698.

[5] JI X. (2012). Research on Fast Image Defogging and Visibility Restoration. Changchun Institute of Optics, Fine Mechanics and Physics, Chinese Academy of Science, Changchun (pp. 10-15).

[6] Vanmali, A. V., Kelka, S. G., \& Gadre, V. M. (2015). A novel approach for image dehazing combining visible-NIR images. Proceedings of the Fifth National Conference on Computer Vision, Pattern Recognition, Image Processing and Graphics (NCVPRIPG) (pp. 1-4).

[7] Graves, N., \& Newsam, S. (2011). Using visibility cameras to estimate atmospheric light extinction. Applications of Computer Vision, 577-584.

[8] Santra, S., \& Chanda, B. (2016). Day/night unconstrained image dehazing, Proceedings of the 23rd International Conference on Pattern Recognition (ICPR) (pp. 1406-1411).

[9] Land, E. H. (1977). The retinex theory of color vision. Sci. Amer., 237(6), 108-128.

[10] Zhang, Y., Huang, W., Bi, W., \& Gao, G. (2016). Colorful image enhancement algorithm based on guided filter and retinex. Proceedings of the 2016 IEEE International Conference on Signal and Image Processing (ICSIP) (pp. 33-36).

[11] Gambhir, D., \& Manchanda, M. (2015). Wavelet based fusion of fuzzy enhanced images captured under improper illumination. Proceedings of the 2nd International Conference on Signal Processing and 
Integrated Networks (SPIN) (pp. 584-588).

[12] Kaur, S., Bansal, M., \& Bathla, A. K. (2016). Dehazing image using analytical model and color attenuation prior. Proceedings of the 2nd International Conference on Next Generation Computing Technologies (NGCT) (pp. 736-741).

[13] Narasimhan, S. G., \& Nayar, S. K. (2000). Chromatic framework for vision in bad weather. Proceedings of IEEE Conference on Computer Vision and Pattern Recognition: Vol. 1. (pp. 598-605).

[14] Nayar, S. K., \& Narasimhan, S. G. (1999). Vision in bad weather. Proceedings of the Seventh IEEE International Conference on Computer Vision: Vol. 2. (pp. 820-827).

[15] Narasimhan, S. G., \& Nayar, S. K. (2003). Contrast restoration of weather degraded images. IEEE Transactions on Pattern Analysis and Machine Intelligence, 25(6), 713-724.

[16] He, K., Sun, J., \& Tang, X. (2009). Single image haze removal using dark channel prior. Proceedings of IEEE Conference on Computer Vision and Pattern Recognition (pp. 1956-1963).

[17] Gibson, K., Vo, D., \& Nguyen, T. (2010). An investigation in dehazing compressed images and video. OCEANS, 2010, 1-8.

[18] Wang, F., Zhang, A., \& Meng, X. (2014). An improved dark channel prior image defogging method. Chinese High Technology Letters, 24(5), 492-497.

[19] Chen, L., Guo, B., \& Bi, J. (2012). Algorithm of single image fog removal based on joint bilateral filter. Journal of Beijing University of Posts and Telecommunications, 35(4), pp. 19-23.

[20] McCartney, E. J. (1976). Optics of the atmosphere: Scattering by molecules and particles. Journal of Modern Optics, 24(7), 779.

[21] Tan, R. T. (2008). Visibility in bad weather from a single image. Proceedings in IEEE Conference on Computer Vision and Pattern Recognition (CVPR) (pp. 1-8).

[22] Ancuti, C., Ancuti, C., Hermans, C., \& Bekaert, P. (2010). A fast semi-inverse approach to detect and remove the haze from a single image. Proceedings of the 10th Asian Conference on Computer Vision, ACCV 2010 (pp. 501-514).

[23] Lai, Y. S., Chen, Y. L., \& Hsu, C. T. (2012). Single image dehazing with optimal transmission map, Proceedings of the 21st International Conference on Pattern Recognition (ICPR2012) (pp. 388-391).

[24] Jobson, D. J., Rahman, Z., \& Woodell, G. A. (1997). Properties and performance of a center/surround retinex. IEEE Transactions on Image Processing, 6(3), 451-462.

[25] Jobson, D. J., Rahman, Z., \& Woodell, G. A. (1997). A multiscale retinex for bridging the gap between color images and the, human observation of scenes. IEEE Transactions on Image Processing, 6(7), 965-976.

[26] Narasimhan, S. G., \& Nayar, S. K. (2003). Shedding light on the weather. Proceedings of the IEEE Computer Society Conference on Computer Vision and Pattern Recognition: Vol. 1. (pp. 665-672).

[27] Tarel, J. P., \& Hautière, N. (2009). Fast visibility restoration from a single color or gray level image. Proceedings of 12th IEEE International Conference on Computer Vision (pp. 2201-2208).

[28] He, K., Sun, J., Tang, X. (2011). Single image haze removal using dark channel prior. IEEE Transactions on Pattern Analysis and Machine Intelligence, 33(12), 2341-2353.

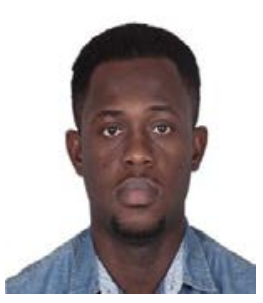

Prince Owusu-Agyeman received the B.Eng. degree in computer science and technology and M.Eng. electrical and computer engineering from the College of Automation Science and Engineering, South China University of Technology in 2013 and 2015, respectively.

He joined the College of Automation Science and Engineering, South China University of Technology in 2015, as a PhD. candidate. His current research interests include computer vision, image processing and real-time image enhancement. 


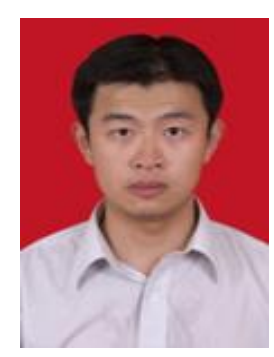

Wei Xie received the B. Eng. and M. Eng. degrees, both in Automation Department, from the Wuhan University of Science and Technology, China, in 1996 and 1999, respectively, and the Ph.D. degree from Kitami Institute of Technology, Japan, in 2003.

He worked as a postdoctoral researcher at the Satellite Venture Business Labortary, from 2003 to 2006. In 2006, he joined the College of Automation Science and Engineering, South China University of Technology, as an associate professor and was promoted to be a full professor in 2010. His research interests include control theory and application. He has published more than 50 papers in international journals and conferences. He is an inventor/co-inventor of 4 China patents. He is a member of the IEEE and has been a reviewer for several prestigious international journals (IEEE Transaction on Automatic control, IET Control Theory \& Applications, International Journal of Control., etc.) and conferences (ACC, CCC, etc.). Research interests and Prof. Xie's expertise areas are control theory and application, pattern recognition and intelligent system techniques.

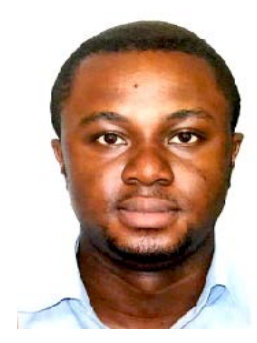

Yao Yeboah received the B.Eng. in the field of electronic information engineering from the Huazhong University of Science and Technology, Wuhan, China in 2011 and the M.Eng. from the South China University of Technology, Guangzhou, China in 2013 in the field of electrical and computer engineering. He subsequently received his Ph.D. in pattern recognition and intelligent systems in 2017 from South China University of Technology. Over the years, he has authored and co-authored several high-level publications sharing research contributions in machine learning, robotic vision and real-time visual perception. In 2017, he joined the School of Automation Science and Engineering, Guangdong University of Technology, as a postdoctoral research fellow. Dr. Yeboah's current research interests include deep learning techniques for autonomous robotics and pattern recognition for real-time applications. 